\title{
ANDES

\section{Respuesta carta al editor titulada: Instrumentos breves: Un método para validar el contenido de los ítems}

\section{Response to the letter of editor entitled: Brief Instruments: A method to validate item content}

\author{
Andry Yasmid Mera-Mamiána, José Andrés Calvache ${ }^{\mathrm{b}}$, Mario Delgado-Noguera ${ }^{\mathrm{b}}$
}

aniversidad CES. Medellín, Colombia

bUniversidad del Cauca. Popoyán, Colombia

\section{Sr. Editor}

Consideramos muy valiosa la propuesta de validación de contenido de la versión final para instrumentos breves, definidos como aquellos instrumentos con diez o menos ítems ${ }^{1}$.

Si bien el instrumento para medir conocimientos de madres y cuidadores sobre alimentación complementaria de infantes ${ }^{2}$ fue sometido a un juicio de expertos, mediante evaluación por 12 médicos especialistas en pediatría quienes evaluaron para cada uno de los ítems: vocabulario, claridad, relevancia y actualidad, no se evaluó la suficiencia de la versión final. Por tanto, consideramos útil la realización de un análisis complementario, utilizando la estrategia de análisis temático ${ }^{3}$, propuesta por los autores de la carta. Los procesos de validación de instrumentos están sujetos a mejoramiento, ante todo considerando la complejidad de los constructos subyacentes a cada temática. La estrategia comentada permite -en nuestro juicio- mejorar el proceso metodológico general ya que adiciona la posibilidad de reproducir, codificar y sistematizar el conocimiento previo que se tiene al respecto de un tópico en particular. De cierta forma, adiciona elementos de síntesis de la evidencia disponible (métodos transparentes y reproducibles) para la construcción de instrumentos breves más sólidos y metodológicamente robustos ${ }^{4}$.
La Tabla 1 presenta el análisis realizado, siguiendo las recomendaciones.

El análisis realizado permite identificar que los ítems que resultaron en la construcción del instrumento para medir conocimientos de madres y cuidadores sobre alimentación complementaria de infantes ${ }^{2}$, incluye la totalidad de ítems que resultan a partir de los procesos de segmentación y categorización.

$\mathrm{Al}$ incluir todas las preguntas del instrumento, para revisar correspondencia con las categorías resultantes, se identifican otros ítems como consistencia, sistema de suministro, lugar, forma y cantidad; dominios que, aunque no se encuentran explícitamente en las definiciones, si son considerados relevantes al hablar de Alimentación complementaria $(\mathrm{AC})^{5-7}$. Adicionalmente, durante la búsqueda de literatura en la etapa de familiarización se identificó que dominios como la seguridad durante la AC, deberán ser tenidos en cuenta en versiones posteriores ${ }^{8}$.

Finalmente, frente al comentario de la carta: "Esta tendencia está motivada por la necesidad de contar con instrumentos que permitan una rápida exploración del niño evitando la fatiga de responder muchas preguntas", es importante aclarar que el instrumento fue diseñado para ser respondido por madres o cuidadores y no por los infantes. 
Tabla 1. Cuadro comparativo de tres definiciones de alimentación complementaria

FAMILIARIZACIÓN Y SEGMENTACIÓN

Organización Mundial de la salud (OMS)/Organización Panamericana de la salud (OPS) ${ }^{5}$

Proceso que comienza cuando la leche maternal sola ya no es suficiente para cubrir las necesidades nutricionales de los lactantes y, por ende, otros alimentos y.líquidos sonn neceșarioș además de la leche materna. El rango de edad óptimo para dar alimentación complementaria está habitualmente entre los 6 - y 24 m meses de. edad.

\section{CATEGORIZACIÓN}

a) Función general (cubrir los requerimientos nutricionales que deja de cumplir la leche materna)

b) Tiempos de inicio y finalización (6 meses a 2 años)

c) Tipo de alimentos (sólidos y líquidos)

d) Función respecto a la leche materna (los complementa, no la reemplaza)

\section{CORRESPONDENCIA}

1. Definición de alimentación complementaria (tipo de alimentons)

2. Edad del niño(a) hasta la que se debe dar leche materna como único alimento (tiempo de in incion)

3. Se debe suministrar suplementos nutricionales o multivitamínicos (tipo de alimentos

4. Consistencia de la comida del niño(a) según sus meses de edad (consistencia*)

5. Sistema adecuado para suministrar alimentos diferentes a la leche materna (sistema de suministro*)

6. Lugar donde se deben dar los alimentos a el/la niño(a) (lugar*)

7. Alimentos para evitar que el/la niño(a) tenga anemia (función general)

8. Edad en que finaliza la alimentación complementaria (tiempo de fiñaliżación)

9. Forma adecuada de dar alimentos complementarios (forma*)

10. Cantidad de alimentos complementarios que se deben suministrar a los 12 meses (cantidad*)

Nota: El subrayado denota los aspectos relevantes en cada definición, mientras el tipo de subrayado permite identificar coincidencias entre las definiciones. Los ítems marcados con asterisco $\left(^{*}\right)$, corresponden a aspectos adicionales incluidos en las preguntas.

\section{Referencias}

1. Ziegler M, Kemper CJ, Kruyen P. Short Scales-Five Misunderstandings and Ways to Overcome Them. Journal of Individual Differences. 1 de enero de 2014;35(4):1859.

2. Sierra-Zúñiga M, Holguin Betancourt C, Mera-Mamián AY, et al. Construcción y validación de un instrumento para medir conocimientos de madres y cuidadores sobre alimentación complementaria de infantes. Revista chilena de pediatría [Internet]. 2020;91(5):711-21. Disponible en: https://scielo.conicyt.cl/scielo. php?script $=$ sci_abstract\&pid $=$ S037041062020000500711\&lng=es\&nrm=iso\&t lng=es

3. Braun V, Clarke V. Using thematic analysis in psychology. Qualitative research in psychology. 2006;3(2): 77-101.

4. Boateng GO, Neilands TB, Frongillo EA, Melgar-Quiñonez HR, Young SL. Best Practices for Developing and Validating Scales for Health, Social, and Behavioral Research: A Primer. Front Public Health. 2018;6:149.

5. Organización Mundial de Salud (OMS), Organización Panamericana de la Salud (OPS). Principios de Orientación para la Alimentación Complementaria del Niño amamantado [Internet]. 2003. Disponible en: https://iris.paho.org/ handle/10665.2/49259

6. Comité de Lactancia Materna y Comité de Nutrición de la Asociación Española de Pediatría. Recomendaciones de la Asociación Española de Pediatría sobre alimentación complementaria [Internet]. 2018. Disponible en: https:// www.aeped.es/sites/default/files/ documentos/recomendaciones_aep_ sobre_alimentacio_n_complementaria_ nov2018_v3_final.pdf

7. Romero-Velarde E, VillalpandoCarrión S, Pérez-Lizaur AB, et al. Consenso para las prácticas de alimentación complementaria en lactantes sanos. Boletín médico del Hospital Infantil de México. 2016;73(5):338-56.

8. World Health Organization (WHO) Complementary feeding [Internet]. Disponible en: https://www.who. int/health-topics/complementaryfeeding\#tab=tab_1 